\title{
In Defense of Heritable Human Genome Editing: \\ On the Geneva Statement by Andorno et al.
}

Tess Johnson

Faculty of Philosophy, University of Oxford

Andorno and colleagues' paper recently published in Trends in Biotechnology condemned support for heritable human genome editing (HHGE) that is claimed to be premature and to have occurred without sufficient public consultation. [1] The general message of the paper is welcome in its emphasis on the importance of gaining broader perspectives on uses and regulation of HHGE before calls for clinical use are made. Yet some problematic arguments for their position lead them to seemingly condemn not only current premature calls for clinical use of HHGE, but any analysis of the ways in which it might be appropriately regulated in the future.

Ethicists and scientists have advocated for proceeding with HHGE trials, if and when it is deemed safe. [2] If this is possible, then rather than being unprepared for regulating a new, disruptive technology, we must conduct further ethical investigation now. The authors claim that any moves to proceed with HHGE would be "abandoning the restraint urged by the United Nations General Assembly". [1] This may be so, but it would also be irresponsible to neglect to explore how the technology could be ethically used and regulated, especially given recent evidence of its inappropriate use. [3] Jiankui He's example should not be used to encourage an indefinite moratorium on HGGE or discourage further ethical discussion. Rather, it highlights the necessity of anticipating the spread of HHGE with adequate, ethically-guided policy, avoiding further inappropriate uses.

In particular, some points made in this article against even considering how we may proceed with HHGE in the future face strong objections. 
Firstly, the authors note HHGE does not prevent disease in any existing person, and this is used as an argument against possible medical benefits that may provide reason to support HHGE. However, HHGE can prevent disease in a future person. Prospective parents who are both carriers for severe autosomal recessive conditions are at risk of having a child with the condition, and some heterozygote-homozygote couples with autosomal dominant conditions would be highly unlikely to have an unaffected child. The authors present genetic selection as a preferable alternative to HHGE, however in the cases above, selection may not offer an alternative. If they wish to have a genetically related child, HHGE presents the only way for these couples, and does provide medical benefit for the future person that an embryo will become. This is the situation of fair comparison when describing disease prevention: either an inevitably affected child is born, or if the embryo is edited, an unaffected child is born. The timing of the edit as occurring before the embryo is termed a person as such makes no moral difference because the benefit occurring is the same for the future person. Against this argument, one may question an assumption that parents should value having children that are genetically related to them. However, certainly if societal consensus ought to influence our analysis as the authors claim, it's clear that many parents currently do hold genetic relatedness as valuable and relevant to debate surrounding HHGE.

A second objectionable argument made in the paper is based upon the assumption that eugenics is intrinsically morally unacceptable, posing an argument against pursuing HGGE. However, when not used as a normatively-loaded term, eugenics is simply the improvement of the human gene pool. $[4,5]$ Improving the human gene pool is not in itself problematic. Certainly, the processes and values involved in eugenic practice in the past have been indefensible, resulting in discrimination, involuntary sterilisation, or outright genocide. However, these are characteristics of the regimes that encouraged and enforced eugenic practices, not part of the intrinsic nature of gene pool improvement itself. HHGE, if voluntary and based on non-discriminatory, pluralist value conceptions, may present an acceptable form 
of human improvement leading to significant good for people both as individuals and as a society. 'Eugenics' by itself is simply not a valid argument against HHGE.

Finally, the authors tacitly assume that HHGE will be privately accessible via the free market. Availability via the free market raises issues of equality of access to HHGE and competitive pressures for parents wishing to advantage their children. However, again, this cannot simply be assumed as the direction that HHGE policy would take. The case is stronger for a wellregulated, restricted market, or state-supported HHGE. [6] This reduces or eliminates many of the justice-related risks raised in the article, including problematic marketing appeals by private fertility clinics, commercial incentives, or emerging class differences between edited and unedited future individuals.

I by no means wish to deny the central message of this paper, that public involvement in ethical analysis of HHGE is essential. However, some of the arguments presented in Andorno and colleagues' work against HHGE more generally fail, upon closer examination, and do not serve the proposed aim of facilitating well-informed public discourse around HHGE.

\section{References}

1. Andorno, R., Baylis, F., Darnovsky, M., et al. (2020). Geneva Statement on Heritable Human Genome Editing: The need for course correction. Trends in Biotechnology 38(4): $351-354$

2. National Academies of Sciences Engineering and Medicine (US) (2017). Human Genome Editing: Science, Ethics and Governance. Washington, D.C.: The National Academies Press. 
3. He, J. (2018). About Lulu and Nana: Twin girls born healthy after gene surgery as singlecell embryos. Accessed April 2, 2020. Available at: https://www.youtube.com/watch? $\mathrm{v}=$ th0vnOmFltc $\& \mathrm{t}=5 \mathrm{~s} \& \mathrm{ab} \_$channel $=$TheHeLab.

4. Agar, N. (2004). Liberal Eugenics : In defence of human enhancement. Oxford: Blackwell Publishing.

5. Anomaly, J. (2018). Defending Eugenics: From cryptic choice to conscious selection. Monash Bioethics Review 35: 24-35.

6. Savulescu, J. (2006). Justice, Fairness, and Enhancement. Annals of the New York Academy of Sciences 1093: 321-338. 